\title{
Decision Making on Pareto Front Approximations with Inherent Nondominance
}

\author{
Markus Hartikainen, Kaisa Miettinen, and Margaret M. Wiecek
}

\begin{abstract}
Approximating the Pareto fronts of nonlinear multiobjective optimization problems is considered and a property called inherent nondominance is proposed for such approximations. It is shown that an approximation having the above property can be explored by interactively solving a multiobjective optimization problem related to it. This exploration can be performed with available interactive multiobjective optimization methods. The ideas presented are especially useful in solving computationally expensive multiobjective optimization problems with costly function value evaluations.
\end{abstract}

\section{Introduction}

In multiobjective optimization (MO), the problem is to find a preferred solution in the presence of several conflicting objectives (see e.g., [7, 17, 23]). In such problems, there is no single well-defined optimal solution but several mathematically equally good solutions, so-called Pareto optimal (PO) solutions, can be identified. To be able to find the most preferred among them as the final solution usually additional preference information is needed from a human decision maker (DM), who knows the problem domain.

According to [7], MO methods can be divided into no-preference methods, a priori methods, a posteriori methods and interactive methods. For details of these different methods see e.g., [17]. Interactive methods can be seen as the most prominent - as argued in [20] - because the DM can learn about the problem as e.g., in [4] and adjust his/her goals accordingly. Because only such PO solutions are generated that are interesting to the DM, interactive methods are generally computationally much less expensive than, for example, a posteriori methods, for which many PO

Markus Hartikainen · Kaisa Miettinen · Margaret M. Wiecek*

Department of Mathematical Information Technology, University of Jyväskylä, Finland

* On sabbatical leave from Clemson University, South Carolina, USA 
solutions must be computed in order for the DM to get enough alternatives for making an informed decision.

If a multiobjective optimization problem (MOP) is computationally very demanding i.e., function evaluations take a lot of time, even the classical interactive methods can have problems; the DM may get frustrated in waiting while new PO solutions that reflect his/her preferences are computed. This high computational demand occurs often, for example, with the so-called black-box MOPs, whose objective functions are not explicitly known as algebraic functions of decision variables but need to be e.g., simulated. Approaches based on evolutionary multiobjective optimization methods have been proposed e.g. in [11, 14] for finding PO solutions for computationally expensive MOPs. Another way to handle such MOPs is to use design of experiments for constructing surrogate approximations of the objective functions and then solving the surrogate MOP [26, 25]. However, according to [9], the use of surrogate approximations loses its computational benefits as the number of objectives increases. The approaches based on evolutionary algorithms have their issues too; in order to produce a dense Pareto set representation, a large number of solutions must be computed, which is time-consuming or sometimes even impossible. On the other hand, the choice of the final outcome from a large number of PO outcomes is cognitively very demanding as noted in [13].

Instead of estimating each objective function with a surrogate, one can estimate the set of PO outcomes (also called the Pareto front (PF)). In [4], an interactive method called Pareto Navigator is proposed, in which a PF approximation for a convex MOP is constructed based on a relatively small set of PO outcomes. Having constructed the approximation, the preferences of the DM can be reflected in real time by moving on the approximation in a way that resembles the visual interactive Pareto Race method for linear problems [12]. When a preferred point on the approximation has been found, the closest actual PO outcome can be found by means of the achievement scalarizing function [24].

The construction of a PF approximation (that allows consideration of the objective vectors between known PO outcomes) and its exploration with $\mathrm{MO}$ methods is a novel approach to computationally demanding MOPs and has some potential benefits. When compared to a posteriori methods, some computational expense can be avoided because a smaller number of PO outcomes is sufficient. Since the DM is allowed to explore the approximation by iteratively expressing his/her preferences, the cognitive load of going through a large set of PO outcomes becomes lighter. The DM can also learn about the problem in the process. Finally, since the construction of the PF approximation does not have any special requirements for the initial PO outcomes, they may be generated with any method for finding PO outcomes, and because the DM is not involved in this, the possible computational cost is not a problem.

The Pareto Navigator method is, however, only applicable to convex MOPs, because of the type of approximation it uses. The ultimate goal behind our study is to develop a MO method that uses the Pareto Navigator's idea of navigating on the approximation for general continuous nonlinear MOPs. The aim of this paper is to concentrate on the issue of what kind of approximation can be used for the above 
mentioned exploration. Because interactive $\mathrm{MO}$ methods have been proven effective in finding a preferred point on the PF, we want to employ them also for finding a preferred point on the approximation. The process of finding a preferred point on a $\mathrm{PF}$ approximation (by using interactive MO methods or other methods) is referred to in this paper as decision making on the approximation.

There are numerous methods for approximating the PF of a MOP. A survey [22] summarizes methods published before 2003. More recent approximation methods for nonlinear MOPs are given e.g., in [3, 5, 15, 16]. However, the uses of the PF approximations in decision making have not received the attention that they deserve. Among the few exceptions are $[2,4,8,10,15,18,21]$. None of these, however, aims at using general interactive MO methods in exploring the PF approximation. The approach of this paper is, thus, different from all the above; we aim at characterizing an approximation for a specific use of exploration with interactive MO methods.

The paper is structured as follows. Some terminology and notation is set in Section 2. We introduce a new property called inherent nondominance (IND) for a PF approximation and prove its relevant properties in Section 3. In Section 4, decision making on the approximation is discussed and an example is given. We also propose an approach to exploring an IND PF approximation by solving a special type MOP. Finally, we draw conclusions in Section 5.

\section{Notation and definitions}

In this paper we study multiobjective optimization problems (MOPs)

$$
\min _{\text {s.t. } x \in X}\left(f_{1}(x), \ldots, f_{k}(x)\right) \text {, }
$$

where $f_{i}: X \rightarrow \mathbb{R}$ is a function for all $i \in\{1, \ldots, k\}, X \subset \mathbb{R}^{n}$ and $k, n \in \mathbb{N}$. We also define a vector valued function $f: X \rightarrow \mathbb{R}^{k}, f(x)=\left(f_{1}(x), \ldots, f_{k}(x)\right)^{T}$ for all $x \in X$.

The set $X$ is called the feasible decision set of (1) and every $x \in X$ is called a (feasible) decision. The set $f(X)$ is the feasible outcome set of (1) and every $z \in f(X)$ is called a (feasible) outcome. The set $\mathbb{R}^{k}$ is called the objective space of (1) and every point $z \in \mathbb{R}^{k}$ is called an objective vector. With the above notation, the MOP (1) can be formulated as

$$
\min _{\text {s.t. } z \in f(X)} z \text {. }
$$

For any objective vectors $z^{1}, z^{2} \in \mathbb{R}^{k}$ notation $z^{1} \leq z^{2}$ means that $z_{i}^{1} \leq z_{i}^{2}$ for all $i=1, \ldots, k$ and $z^{1} \neq z^{2}$. If $z^{1} \leq z^{2}$ it is said that $z^{1}$ dominates $z^{2}$. If $B \subset \mathbb{R}^{k}$ is a set, then a point $b \in B$ is Pareto optimal (PO) in $B$, if there does not exist a point $\tilde{b} \in B$ that dominates $b$. An outcome $z \in f(X)$ is PO for (1), if $z$ is PO in $f(X)$. Finally, the Pareto front (PF) of B, $P F(B)$, is the set of PO points in $B$ and the PF of a MOP, $P F$, is the set of PO outcomes of the MOP. A feasible decision $x \in X$ is a PO solution to (1), if $f(x)$ is a PO outcome. 
We assume a rational decision maker (DM): he/she always prefers a PO objective vector over a dominated one in any set of possible objective vectors. In other words, for every set $A \subset \mathbb{R}^{k}$ the preferred objective vectors of the DM belong to $P F(A)$ and, furthermore, the DM is not interested in $A \backslash P F(A)$ if he/she is aware of $P F(A)$.

The starting point of this study is as follows. Assume that we are given a computationally demanding MOP with the above notation. This problem is referred to as the initial MOP. If not otherwise defined, the notations PO and PF always refer to this problem. We also assume that we are given a finite set of $\mathrm{PO}$ outcomes $P$. This set is referred to as the initial set of PO outcomes. As in Pareto Navigator, the way this set has been generated is not restricted in any way.

\section{Inherent nondominance and inherently nondominated approximation of the $\mathbf{P F}$}

In this section we introduce the concept of inherent nondominance and other related concepts. We also present the relevant properties characteristic for an inherently nondominated approximation of the PF. These properties will be further interpreted in Section 4.

Definition 1. We say that a set $A \subset \mathbb{R}^{k}$ is inherently nondominated (IND), if for any two points $a^{1}, a^{2} \in A$ neither $a^{1} \leq a^{2}$ nor $a^{2} \leq a^{1}$.

Definition 2. A set $A \subset \mathbb{R}^{k}$ is an inherently nondominated $P F$ approximation (based on $P$ ) if $A$ is IND and $P \subset A$.

According to the following theorem, the actual PF satisfies the IND condition. The actual PF should be suitable as an approximation of itself, because naturally it is the best approximation that one can hope for.

Theorem 1. The PF is an IND PF approximation based on $P$.

Proof. Since $P$ is a set of $\mathrm{PO}$ outcomes, then $P \subset P F$. The property that $P F$ is IND is trivial, since if $z^{1}, z^{2} \in f(X)$ so that $z^{1} \leq z^{2}$, then $z^{2} \notin P F$.

The following theorem gives a sufficient and necessary condition for a set to be IND and could, thus, have been chosen as the definition for an IND set. We, however, chose the definition given above.

Theorem 2. Let $A \subset \mathbb{R}^{k}$ be a set. Then the set $A$ is IND if and only if $P F(A)=A$.

Proof. " $\Rightarrow$ ": Naturally $P F(A) \subset A$. To show that $A \subset P F(A)$, assume the contrary that there exists a point $a \in A \backslash P F(A)$. This implies a point $b \in A$ so that $b \leq a$. But this is a contradiction, since $A$ is IND.

$" \Leftarrow "$ : To show that $A$ is IND assume the contrary that there exist distinct points $a, b \in A$ so that $a \leq b$. This would imply that $b \notin P F(A)$ and would thus be a contradiction. 
The following corollary follows directly from Theorem 2 . It is, however, considerably important, since according to it an IND approximation extends $P$ without losing Pareto optimality.

Corollary 1. If a set $A \subset \mathbb{R}^{k}$ is an IND PF approximation based on $P$, then

$$
P \subset P F(A)
$$

Proof. Now $P F(A)=A \supset P$, because of Theorem 2 and Definition 2.

The above properties of an IND approximation are rather straightforward. Theorem 3 establishes the existence of IND PF approximations.

Theorem 3. The set of initial PO outcomes $P$ is an IND PF approximation based on $P$.

Proof. Because outcomes in $P$ are $\mathrm{PO}$, they do not dominate each other. Also $P \subset P$ trivially.

The IND approximation $P$ is not, however, interesting, since it does not contain any points outside the set of initial PO outcomes $P$. We want to construct IND approximations $A$ that extend $P$, but we must skip the development of algorithms producing such IND approximations because of space limitions. Our general idea is to start with a large set $B, P \subset B \subset \mathbb{R}^{k}$, and then remove from $B$ such points $b \in B \backslash P$ that violate the IND condition. By Theorem 3 , this procedure produces an IND approximation. Also by Zorn's lemma, there exists at least one minimal (by inclusion) set $\tilde{B} \subset B \backslash P$ such that $B \backslash \tilde{B}$ is an IND PF approximation.

Example 1. Let the set of initial PO points be $P=\left\{p^{1}, p^{2}, p^{3}, p^{4}\right\}$, where $p^{1}=$ $(1,0,0), p^{2}=(0,1,0), p^{3}=(0,0,1)$ and $p^{4}=(2 / 5,2 / 5,2 / 5)$. We compare two piecewise linear PF approximations $A^{1}=\operatorname{conv}(P)$ and $A^{2}=\operatorname{conv}\left(p^{1}, p^{2}, p^{4}\right) \cup$ $\operatorname{conv}\left(p^{1}, p^{3}, p^{4}\right) \cup \operatorname{conv}\left(p^{2}, p^{3}, p^{4}\right)$, where $\operatorname{conv}(C)$ is the convex hull of a set $C$. These are shown in Figure 1. The convex hull of all outcomes in $P$ is used e.g., in $[4,21]$ for approximating the outcome set $f(X)$ for convex MOPs and has thus been used for getting also a PF approximation. Approximation $A^{2}$ is IND, which can be shown analytically. Approximation $A^{1}$ is, however, not IND because the point $(1 / 3,1 / 3,1 / 3) \in A^{1}$ dominates the initial PO outcome $p^{4}$ that is also in $A^{1}$. For this reason, approximation $A^{1}$ is not able to capture the non-convex shape implied by the initial set of PO outcomes and, unlike the IND approximation $A^{2}$, gives too optimistic estimates.

\section{Decision making on the approximation}

In this section we discuss decision making on the approximation. The underlying reasoning is that once the PF approximation with desired properties has been created, one can navigate on it with significantly lower computational cost than on the 
Objective 2

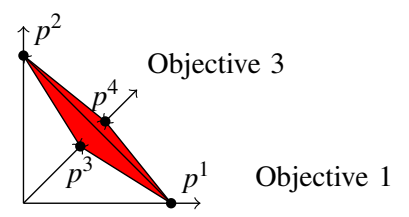

(a) Approximation $A^{1}$
Objective 2

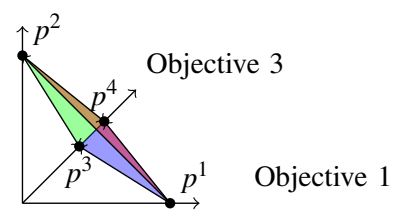

(b) Approximation $A^{2}$

Fig. 1: Two piecewise linear PF approximations constructed with the same initial set of PO outcomes. The approximation $A^{1}$ is the convex hull of all points and the approximation $A^{2}$ is a union of three triangles.

PF of the original problem. Once a desired objective vector on the approximation has been found, one can generate the closest corresponding PO solution as in Pareto Navigator.

The problem of finding a preferred point on a PF approximation is similar to finding a preferred outcome of a MOP; like the PF, its approximation $A$ may have many mathematically equally good objective vectors and, thus, there is a need for decision making even on the PF approximation. An important aspect of decision making on an approximation is the question of how well the preferences of the DM on the approximation reflect the preferences on the real PF.

Based on a limited knowledge of the PF in a form of the initial set of PO outcomes $P$, the accuracy of the approximation cannot naturally be guaranteed outside the initial PO outcomes. What can, however, be done is that a rational DM is given the opportunity to choose any of the initial PO points in $P$ while making rational decisions on the approximation. An undesirable occurrence happens e.g., in non-IND approximation $A^{1}$ in Example 1, where a rational DM will not be interested in the initial PO outcome $p^{4}$, because this point is not $\mathrm{PO}$ on the approximation.

Assuming that the PF approximation discussed above is IND, then we have the following: First of all, all points in the initial set of PO outcomes are now plausible decisions for a rational DM because $P \subset P F(A)$ by Corollary 1 . Second, since $P F(A)=A$ by Theorem 2, the diversity of the PO objective vectors on the approximation can be examined directly from $A$. Since the DM is rational, then the diversity of $P F(A)$, not that of $A$, is interesting to him/her. If the PF approximation $A$ is nonIND, these are not the same things.

We are left with the question of how to help the DM find a preferred point on a PF approximation. This is another instance where the concept of an IND approximation helps. According to Theorem 4, an IND approximation is the PF of a MOP that also has the set $P$ on its PF.

Theorem 4. If a set $A \subset \mathbb{R}^{k}$ is an IND PF approximation based on $P$, then there exists a MOP with notation (1) so that $P \subset P F(f(X))$ and $P F(f(X))=A$. 
Proof. Assume that $f=I$ (the identity mapping), $X=A$ and $n=k$ in (1). Then $P F(f(X))=P F(X)=P F(A)=A$, where the last equation results from Theorem 2. Also by definition $P \subset P F(f(X))=A$.

The MOP, whose existence is guaranteed by Theorem 4, can be seen as a surrogate MOP for the initial MOP. We define this MOP for a general set, and not just for an IND approximation, and also give it a name.

Definition 3. For any nonempty set $A \subset \mathbb{R}^{k}$, the MOP

$$
\min _{\text {s.t. } z \in A} z
$$

is called the MOP implied by $A$. The formulation above is analogous to formulation (2) but instead of the set of outcomes $f(X)$, an arbitrary set $A$ in the objective space $\mathbb{R}^{k}$ is used.

When trying to find a preferred point on an IND PF approximation, we thus propose to solve the MOP implied by $A$ and take the final solution of the former as a preferred point on the approximation $A$. This approach has a couple of apparent benefits: All methods developed for solving MOPs are now applicable for decision making on the approximation and there is no need to develop new ones for this. Especially we can now use interactive MO methods in solving MOP (3). Also, since the PF of the MOP implied by the approximation is explicitly known, the computational expense should be very low while using MO methods in solving this MOP. Furthermore, since all the outcomes of the MOP are actually already on the PF of this MOP, there is no need to be concerned about the convergence to the PF, which also saves computational time. These aspects are clarified below with an example.

Example 2. In [6], a computationally expensive, black-box problem of wastewater treatment planning involving three objective functions is solved with the interactive multiobjective method NIMBUS [19, 17]. In the optimization process, $11 \mathrm{PO}$ outcomes including the final preferred outcome $(0.72,332,524)^{T}$ were computed. Here we want to produce a PF approximation in the neighborhood of the final outcome and then to formulate a MOP implied by this approximation in a form that could be solved with interactive MO methods. We use the already generated PO outcomes to construct this approximation but we want to emphasize that any appropriate method could have been used to generate the set P. We choose a subset of known PO outcomes (and thus approximate only a part of the PF) for the simplicity of the presentation.

The final outcome and four outcomes closest to it are chosen as the initial set of PO outcomes $P$. Thus, we have $P=\left\{p^{1}, p^{2}, p^{3}, p^{4}, p^{5}\right\}$, where $p^{1}=(1.7,326,506)$, $p^{2}=(1.1,336,515), p^{3}=(0.9,333,519), p^{4}=(0.7,332,524), p^{5}=(0.5,347,528)$. The set

$$
A=\operatorname{conv}\left(p^{1}, p^{2}, p^{3}\right) \cup \operatorname{conv}\left(p^{1}, p^{3}, p^{4}\right) \cup \operatorname{conv}\left(p^{3}, p^{4}, p^{5}\right) \cup \operatorname{conv}\left(p^{2}, p^{3}, p^{5}\right)
$$

shown in Figure 2 is an IND PF approximation and this can be checked analytically. 


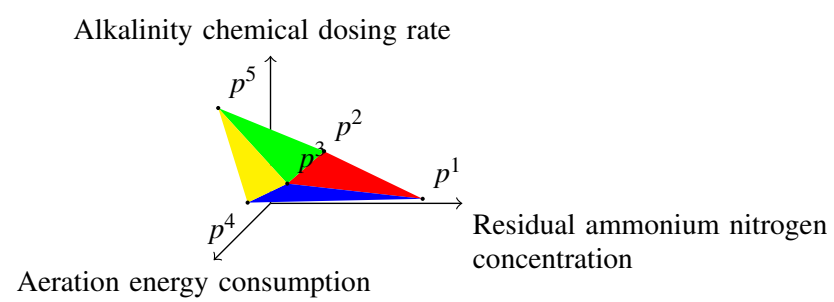

Fig. 2: The IND PF approximation $A$.

The IND PF approximation $A$ can be parametrized with a slack variable $t \in$ $\{0,1\}^{3} \times[0,1]^{2}$ and, using this parametrization, the MOP implied by $A$ can be formulated as

$$
\begin{array}{ll}
\min z(t) & \\
\text { s.t. } z(t) & =t_{1}\left(t_{4} p^{1}+t_{5} p^{2}+\left(1-t_{4}-t_{5}\right) p^{3}\right) \\
& \left.+t_{2}\left(t_{4} p^{1}+t_{5} p^{3}\right)+\left(1-t_{4}-t_{5}\right) p^{4}\right) \\
& +t_{3}\left(t_{4} p^{2}+t_{5} p^{3}+\left(1-t_{4}-t_{5}\right) p^{5}\right) \\
& +\left(1-t_{1}-t_{2}-t_{3}\right)\left(t_{4} p^{3}+t_{5} p^{4}+\left(1-t_{4}-t_{5}\right) p^{5}\right) \\
t= & \left(t_{1}, \ldots, t_{5}\right) \in\{0,1\}^{3} \times[0,1]^{2} \\
0 \leq & 1-t_{1}-t_{2}-t_{3} \text { and } 0 \leq 1-t_{4}-t_{5} .
\end{array}
$$

The MOP (4) includes 2 continuous and 3 discrete decision variables, 2 linear inequalities concerning them and also some box constraints. The decision variable $t$ does not have any meaning to the DM, but is merely a product of the parametrization of $A$. However, the objective function values in the vector $z(t) \in A$ have a meaning to the DM for each feasible $t$ and based on these values he/she can express preferences. Exploration of the PF approximation $A$ means varying the decision variable $t$ based on the preferences of the DM and then showing him/her the resulted objective function values.

MOP (4) can by inputed e.g., to the WWW-NIMBUS implementation of the NIMBUS method [1]. Solving it is computationally inexpensive and the DM can get feedback to his/her preferences quickly unlike with the initial computationally expensive MOP. Thus, the DM could be more inclined to see more new outcomes and this should help him/her obtain a better understanding of the problem.

The ideas presented in this paper are of general nature, but can be seen to be related to some specific MO methods found in the literature. The Pareto Navigator method [4] can be interpreted as solving a MOP implied by the convex cone of the Pareto optimal solutions. The surface of this convex cone approximates the PF since for Pareto Navigator the problem is assumed to be convex. The methods of [15], on the other hand, can be seen as using a visual technique called the Interactive Decision Map for solving the MOP implied by an approximation (that is constructed by methods mentioned therein) of the feasible outcome set. However, since the ap- 
proximations used in these methods are not necessarily IND approximations, the benefits mentioned in this section do not apply.

\section{Conclusion}

We have proposed a new approach to solving computationally expensive multiobjectice optimization problems. In our approach, we first approximate the Pareto front and then enable the decision maker to explore the approximation with his/her preferences. We have also proposed inherent nondominance, a new property for Pareto front approximations which ensures desirable properties for approximations so that exploration can take place.

The utilization of proposed approximation approach requires an algorithm to construct an inherently nondominated Pareto front approximation from a given set of Pareto optimal outcomes. Furthermore, we must study which interactive multiobjective optimization methods are best suited for solving the multiobjective optimization problem implied by the approximation. These issues will be considered in future papers.

Acknowledgements The research was partly supported by the Academy of Finland, grant number 128495 .

\section{References}

1. WWW-NIMBUS System for Multiobjective Optimization, Last accessed: April 15, 2010. http://nimbus.mit.jyu.fi/.

2. H. Ackermann, A. Newman, H. Röglin, and B. Vöcking. Decision-making Based on Approximate and Smoothed Pareto Curves. Theoretical Computer Science, 378:253-270, 2007.

3. V. E. Bezerkin, G. K. Kamenev, and A. V. Lotov. Hybrid Adaptive Methods for Approximating a Nonconvex Multidimensional Pareto Frontier. Computational Mathematics and Mathematical Physics, 46:1918-1931, 2006.

4. P. Eskelinen, K. Miettinen, K. Klamroth, and J. Hakanen. Pareto Navigator for Interactive Nonlinear Multiobjective Optimization. OR Spectrum, 23:211-227, 2010.

5. T. Goel, R. Vaidyanathan, R. T. Haftka, W. Shyy, N. V. Queipo, and K. Tucker. Response Surface Approximation of Pareto Optimal Front in Multi-Objective Optimization. Computer Methods in Applied Mechanics and Engineering, 196:879-893, 2007.

6. J. Hakanen, K. Sahlsted, and K. Miettinen. Simulation-Based Interactive Multiobjective Optimization in Wastewater Treatment. In Proceedings of the International Conference on Engineering Optimization EngOpt 2008, Rio de Janeiro, Brazil, 2008. http: //www . engopt.org/08/nukleo/pdfs/0205_engopt_paper_hakanen.pdf [Last accessed: 19.11.2009].

7. C. Hwang and A. S. M. Masud. Multiple Objective Decision Making - Methods and Applications: a State-of- the-Art Survey. Springer, Berlin, 1979.

8. I. Kaliszewski. Quantitative Pareto Analysis by Cone Separation Technique. Kluwer Academic Publishers, Dordrecht, 1994. 
9. M. K. Karakasis and K. C. Giannakoglou. On the Use of Surrogate Evaluation Models in Multi-Objective Evolutionary Algorithms. In P. Neittaanmäki, T. Rossi, S. Korotov, E. Onate, J. Periaux, and D. Knörzer, editors, ECCOMAS 2004: Proceedings, Jyväskylä, Finland, 2004. http://www.mit. jyu.fi/eccomas2004/proceedings/pdf/616.pdf, [Last accessed: 19.11.2009].

10. K. Klamroth and K. Miettinen. Integrating Approximation and Interactive Decision Making in Multicriteria Optimization. Operations Research, 56:222-234, 2008.

11. J. Knowles. ParEGO: A Hybrid Algorithm with On-Line Landscape Approximation for Expensive Multiobjective Optimization Problems. IEEE Transactions on Evolutionary Computation, 10:50 - 66, 2006.

12. P. Korhonen and J. Wallenius. A Pareto Race. Naval Research Logistics, 35:615-623, 1988.

13. O. I. Larichev. Cognitive Validity in Design of Decision-Aiding Techniques. Journal of MultiCriteria Decision Analysis, 3:127 - 138, 1992.

14. W. Liu, Q. Zhang, E. Tsang, and B. Virginas. Tchebycheff Approximation in Gaussian Process Model Composition for Multi-Objective Expensive Black Box. In 2008 IEEE Congress on Evolutionary Computation, CEC 2008, pages 3060-3065, Hong Kong, China, 2008.

15. A. V. Lotov, V. A. Bushenkov, and G. A. Kamenev. Interactive Decision Maps. Kluwer Academic Publishers, Boston, 2004.

16. J. Martin, C. Bielza, and D. R. Insua. Approximating Nondominated Sets in Continuous Multiobjective Optimization Problems. Naval Research Logistics, 52:469-480, 2005.

17. K. Miettinen. Nonlinear Multiobjective Optimization. Kluwer Academic Publishers, Boston, 1999.

18. K. Miettinen, A. V. Lotov, G. K. Kamenev, and V. E. Bezerkin. Integration of Two Multiobjective Optimization Methods for Nonlinear Problems. Optimization Methods and Software, 18:63-80, 2003.

19. K. Miettinen and M. M. Mäkelä. Synchronous Approach in Interactive Multiobjective Optimization. European Journal of Operational Research, 170:909-922, 2006.

20. K. Miettinen, F. Ruiz, and A. Wierzbicki. Introduction to Multiobjective Optimization: Interactive Approaches. In J. Branke, K. Deb, K. Miettinen, and R. Slowinski, editors, Multiobjective Optimization: Interactive and Evolutionary Approaches, pages 27-57. Springer, Berlin, 2008.

21. M. Monz, K. H. Kufer, T. R. Bortfeld, and C. Thieke. Pareto Navigation - Algorithmic Foundation of Interactive Multi-Criteria IMRT Planning. Physics in Medicine and Biology, 53:985-998, 2008

22. S. Ruzika and M. M. Wiecek. Approximation Methods in Multiobjective Programming. Journal of Optimization Theory and Applications, 126:473-501, 2005.

23. Y. Sawaragi, H. Nakayama, and T. Tanino. Theory of Multiobjective Optimization. Academic Press, Orlando, 1985.

24. A. Wierzbicki. On the Completeness and Constructiveness of Parametric Characterizations to Vector Optimization Problems. OR Spectrum, 8:73-87, 1986.

25. B. Wilson, D. Cappelleri, T. W. Simplson, and M. Frecker. Efficient Pareto Frontier Exploration using Surrogate Approximations. Optimization and Engineering, 2:31-50, 2001.

26. M. Yoon, H. Nakayama, and Y. Yun. Sequential Approximate Multiobjective Optimization Using Computational Intelligence. Springer, Berlin Heidelberg, 2009. 